\title{
Beware of traumatic anosmia in COVID-19 pandemic
}

\author{
Takahiko Nagamine ${ }^{1,2}$ (D)
}

Received: 22 March 2021 / Accepted: 1 April 2021 / Published online: 21 April 2021

(C) The Author(s), under exclusive licence to Canadian Association of Emergency Physicians (CAEP)/ Association Canadienne de Médecine d'Urgence (ACMU) 2021

Keywords Anosmia $\cdot$ COVID-19 pandemic $\cdot$ Minor head trauma

\section{Dear Editor,}

Anosmia is increasingly recognized as one of the most important symptoms of corona virus disease-19 (COVID19). Among patients presenting with influenza-like symptoms in the emergency department (ED), the presence of anosmia could suggest COVID-19 infection. However, anosmia can also be caused by minor head injury.

A 70-year-old man presented to the ED with sudden onset of anosmia, and a 3-day history of dry cough and headache. The chest X-ray showed no abnormalities, and loop-mediated isothermal amplification (LAMP) test for coronavirus was negative. He recently hit the back of his head on the floor. Neurological examination revealed no abnormalities. Head computed tomography (CT) showed no abnormalities (Fig. 1a), but magnetic resonance imaging (MRI) showed brain contusions in the bilateral orbitofrontal lobes on fluidattenuated inversion recovery (FLAIR) images (Fig. 1b). He was diagnosed with post-traumatic anosmia and treated with steroids and Chinese herbal medicine.

Among patients presenting to the ED with influenza-like symptoms, the presence of anosmia was associated with a 14-fold increase in the positivity rate of COVID-19 [1]. The most common symptoms associated with COVID-19 are fever, dyspnea, cough, and anosmia, of which anosmia is reported to have the highest odds ratio of 3.2 [2]. Patients with COVID-19 have a higher rate of anosmia, because coronaviruses can damage the olfactory neuroepithelium by apoptosis, followed by a decrease in mature sensory neurons [3]. However, anosmia also occurs in people without COVID-19 infection. Our case illustrates the need to fully investigate the cause of anosmia and rule out overdiagnosis of COVID-19, especially in patients at high risk for COVID-19. Traumatic anosmia is not properly diagnosed in emergency settings because it can occur in elderly people even with minor head trauma, and the history of trauma may not be known [4]. MRI can play a major role in detecting underlying abnormalities of the olfactory system, ruling out the possibility of anosmia due to COVID-19. It has been reported that a case resembling COVID-19 infection was diagnosed as anosmia due to ischemic olfactory infarction on MRI [5].

In the COVID-19 pandemic, anosmia is an important finding to suspect the presence of this infection. However, anosmia can be caused by a variety of factors, and the possibility of trauma should be considered in the ED. MRI of the orbitofrontal cortex is useful in differentiating between traumatic anosmia and COVID-19 induced anosmia. During an outbreak of COVID-19, a careful differential diagnosis of anosmia is required.

Takahiko Nagamine

tnagamine@outlook.com

1 Sunlight Brain Research Center, 4-13-18 Jiyugaoka, Hofu, Yamaguchi 747-0066, Japan

2 Department of Emergency Medicine, Matsumoto Surgical Hospital, Hofu, Yamaguchi, Japan 

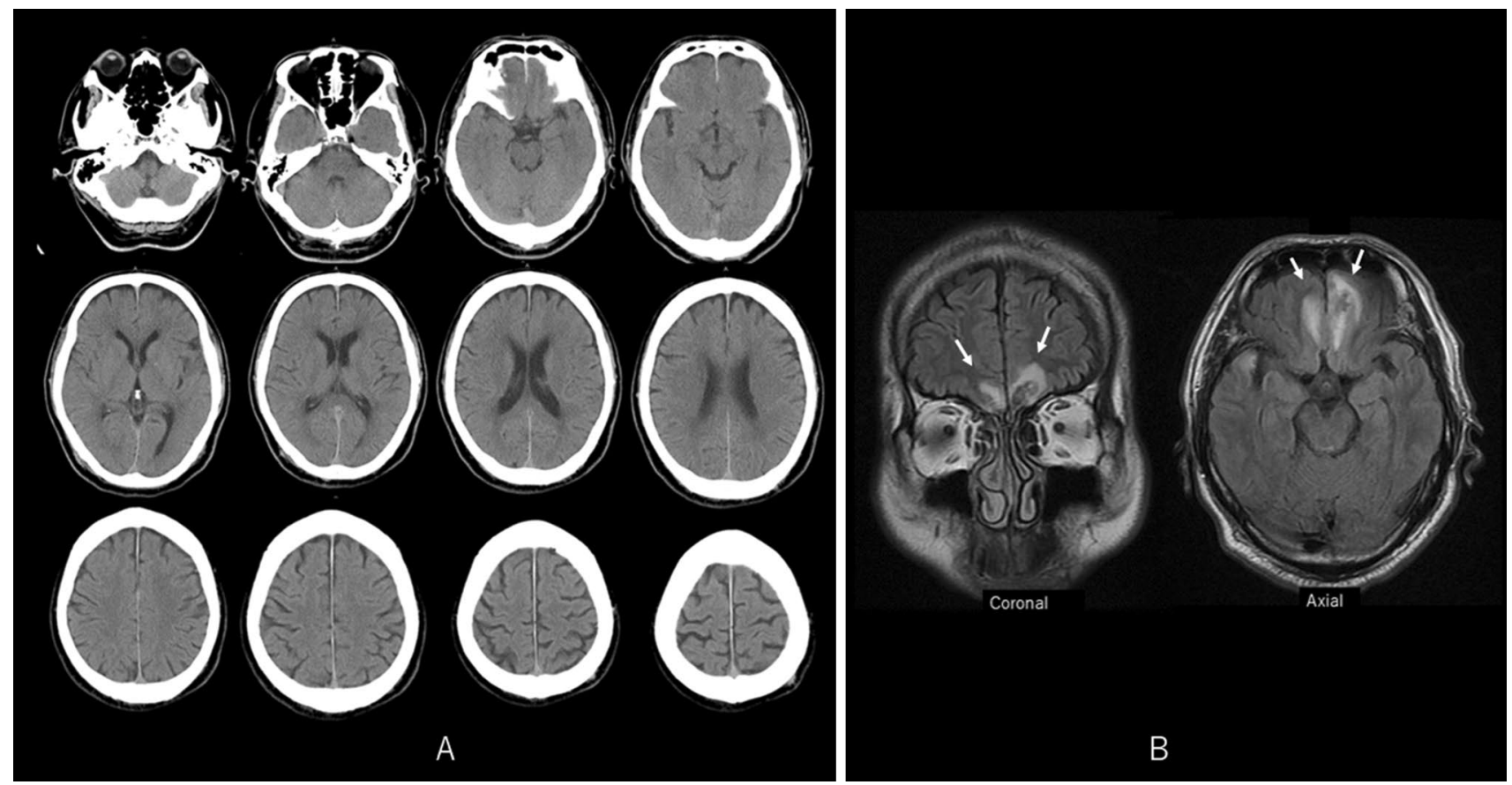

Fig. 1 a Head computed tomography showing no abnormalities. b Fluid-attenuated inversion recovery (FLAIR) images showing brain contusion in bilateral orbitofrontal cortex

\section{Declarations}

Conflict of interest The author reports no conflicts of interest.

\section{References}

1. Lee DJ, Lockwood J, Das P, Wang R, et al. Self-reported anosmia and dysgeusia as key symptoms of coronavirus disease 2019. CJEM. 2020;22(5):595-602.

2. Romero-Gameros CA, Colin-Martínez T, Waizel-Haiat S, et al. Diagnostic accuracy of symptoms as a diagnostic tool for
SARS-CoV 2 infection: a cross-sectional study in a cohort of 2,173 patients. BMC Infect Dis. 2021;21(1):255.

3. Cataldi M, Pignataro G, Taglialatela M. Neurobiology of coronaviruses: potential relevance for COVID-19. Neurobiol Dis. 2020;143:105007.

4. Bratt M, Skandsen T, Hummel T, et al. Frequency and prognostic factors of olfactory dysfunction after traumatic brain injury. Brain Inj. 2018;32(8):1021-7.

5. Theodorou DJ, Theodorou SJ, Tsaggou V, et al. Anosmia caused by ischaemic olfactory infarction: false alert for COVID-19 infection. QJM. 2021;114(1):50-1. 